\title{
A Deep Wide Field Survey From Digitally Stacked Schmidt Plates
}

\author{
M. R. S. Hawkins \\ Royal Observatory, Blackford Hill, Edinburgh EH9 3HJ, UK
}

\begin{abstract}
A large set of exposures of a single UK Schmidt Telescope field has been obtained, covering five colour bands over 18 years. Techniques have been developed for combining digitised data from many plates. The results of adding 64 exposures in $B_{j}$ and $\mathrm{R}$ are demonstrated showing that the expected gain of $\sim 2.5$ magnitudes in limiting magnitude are achieved. By combining various subsets of the data, proper motions and variability can be determined. Two examples of the many possible uses of the dataset are given: the discovery of low mass brown dwarf candidates using colours and proper motions, and of quasars using variability and colours.
\end{abstract}

\section{The Combination of Multiple Exposures}

In 1977 shortly after the UK $1.2 \mathrm{~m}$ Schmidt was commissioned, a programme to monitor variables over as many timescales as possible was started. A single field (ESO/SERC 287) at $21 \mathrm{~h} 28 \mathrm{~m},-45^{\circ}$ was photographed on all timescales from an hour to 18 years in $U B_{j} V R I$ passbands. It was an attractive possibility right from the beginning to stack the plates in some way to achieve greater depth, and in the first instance, the feasibility of a photographic procedure was examined with David Malin of the AAO. There were however a number of difficulties which made the prospect as originally envisaged unfeasible. The main problem was that due to differential refraction between plates taken at different hour angles it was not possible to superimpose the fields exactly. The only solution was to cut the field into 16 squares and add each square separately. Apart from the huge amount of labour involved, there were inevitably edge effects between the squares, and there was no obvious way of calibrating the final result.

A more attractive alternative had always been to add the plates digitally, but until recently the available computing facilities were not adequate for such a task. This is now no longer a problem, and modern machines have the speed and storage to carry out the stacking in a realistic time. The first step in the stacking procedure is to define a master coordinate system from one of the plates, and obtain parameterised data for all the images in the field. This was done by measuring the plate in COSMOS mapping mode which digitises the whole area in $16 \mu \mathrm{m}$ pixels, and then running the COSMOS analyser on this data to obtain image positions. This procedure was then repeated in turn for each of the plates to be stacked. To allow for the different qualities of the plates, a weighting factor is derived. The sky noise, $\sigma_{s}$, is measured in several standard regions, and 
a dozen or so star images fitted to obtain their width at half maximum, $\sigma_{i}$. The weighting factor is then simply $\left(\sigma_{s} \sigma_{i}\right)^{-2}$. Next, a coordinate transformation (rotation, translation and scale change) is evaluated in 15 arcmin squares from the new plate to the master plate, using stars detected by the COSMOS analyser. This transformation is then used to convert each pixel position into the master frame, and the master pixels are increased by an amount obtained by linear interpolation between the nearest four pixels to the transformed position, with the appropriate weighting factor applied. This procedure is then repeated for every plate to be stacked.

The question of what analytical form the pixels should be when they are added required some thought. In principle, if a suitable intensity dependent weighting function is used then the result will be the same for any analytical form. However, the plates are measured in transmission, which is a highly nonlinear representation of the images on the plate, and is not suitable for the addition process. Intensity derived from the calibration spots was a possibility, but the extra work required to calibrate each plate separately, combined with the requirement for an intensity dependent weight made it unattractive. The best answer is to use photographic density, which is simply the logarithm of transmission. This has the advantage of simplicity, combined with the fact that the noise is essentially independent of density level and so a single weighting factor for each plate is sufficient.

Fig. 1a shows a grey scale plot of part of a survey quality plate of the field. Fig. lb shows the result of stacking 64 plates. In Fig. la, the image A near the plate limit has $B_{j}=22$. 5 . In Fig. lb this image is now very well defined, and image $B$ near the stack limit has $B_{j}=25$.

When the addition was complete, the COSMOS analyser was run on the stacked mapping data to produce the standard COSMOS parameter output. This was then calibrated in the ordinary way using deep CCD frames taken with the ESO $3.6 \mathrm{~m}$ at La Silla. Fig. 2 shows a plot of CCD magnitude against the transformed COSMOS measures. The scatter is quite small even out to $\mathrm{B}=25$, demonstrating that in this 64 plate stack the theoretical improvement from the addition process is being achieved. Plates were available for stacking in five passbands - UBVRI, and Table 1 summaries the magnitudes achieved after addition. "No" is number of plates in stack, "Complete" signifies magnitude to which the sample is essentially complete, and "Limiting" the faintest objects which can be detected.

TABLE 1 Plate Stacking in Field 287

\begin{tabular}{|l|l|c|l|l|}
\hline Passband & Emulsion & No. & Complete & Limiting \\
\hline$B_{j}$ & IIIa-F & 65 & 24 & 25.5 \\
\hline R & IIIa-F & 65 & 22.5 & 24 \\
\hline I & IVN & 30 & 20.5 & 22 \\
\hline U & IIIa-J & 7 & 22.5 & 24 \\
\hline V & IIIa-F & 3 & 21 & 22.5 \\
\hline
\end{tabular}



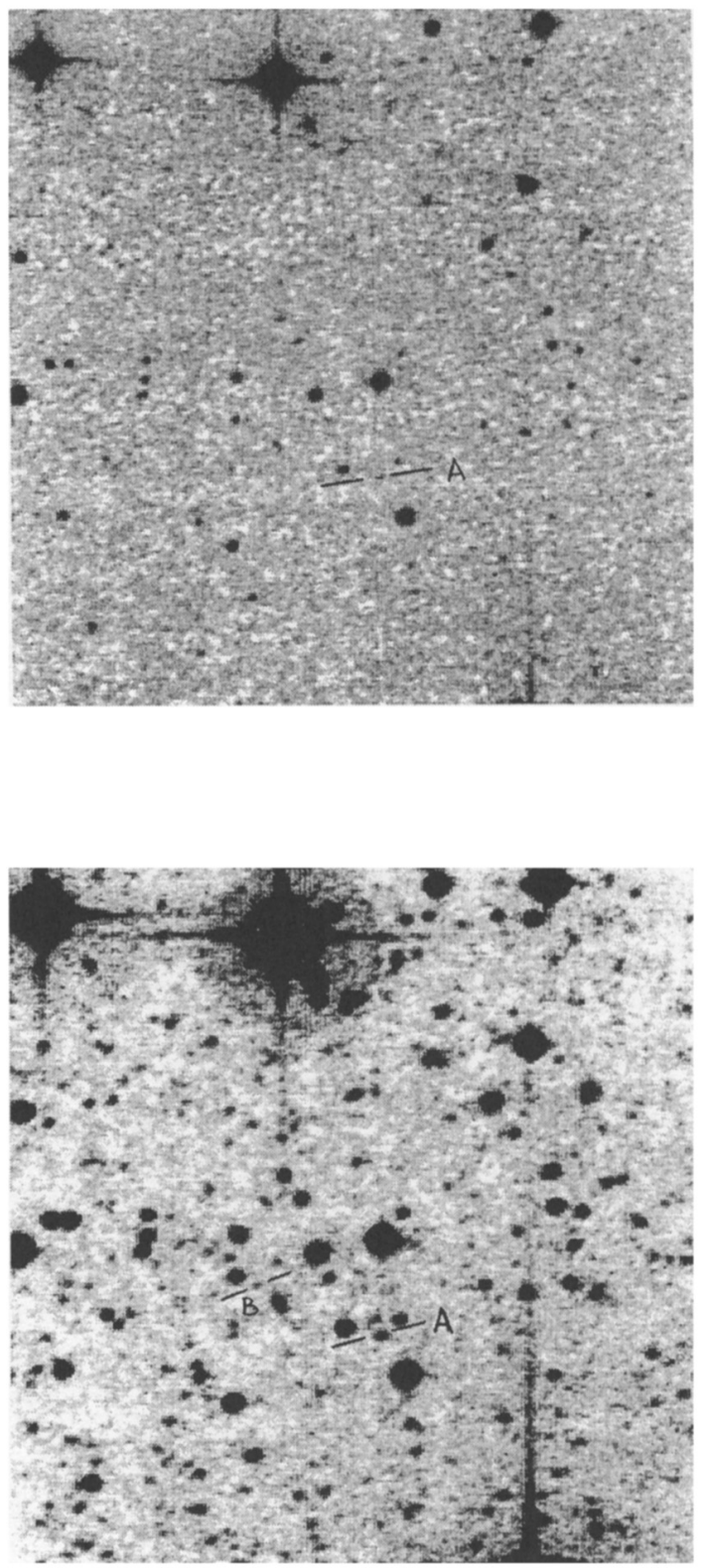

Figure 1. (a) Grey scale plot of COSMOS measures of a 4 arcmin square from a survey quality plate in field 287. (b) The same area of sky from the 64 plate stack of IIIa-J plates. 
Stacked Data

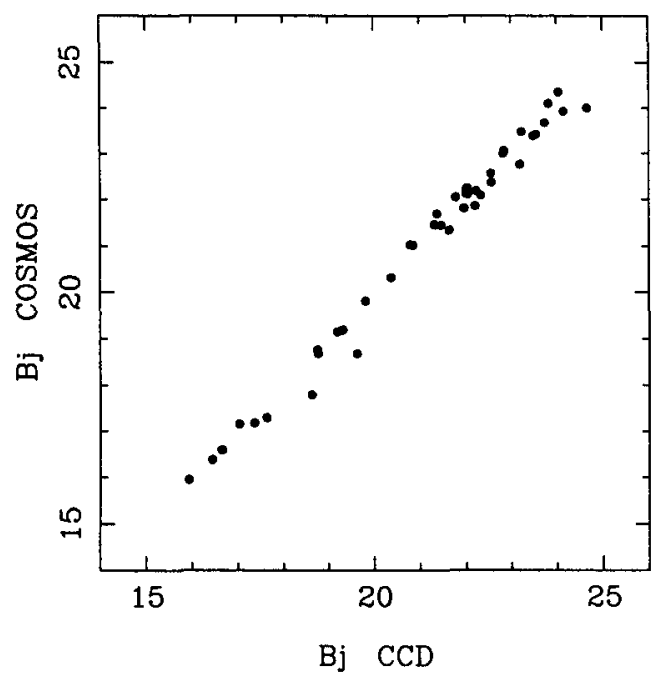

Figure 2. Plot of a deep CCD sequence against transformed COSMOS measures from the stack.

As well as creating the full stacks of 64 plates for the $B_{j}$ and $\mathrm{R}$ passbands, it was also possible to create substacks containing all plates taken in a single year (or several years). Although this procedure inevitably reduced the limiting magnitude, it made possible the measurement of variability and proper motions to significantly greater depth than is achievable on a single plate. Fig. 3 shows a histogram of proper motions for yearly substacks from the $B_{j}$ data. It will be seen that the error is around 20 mas/yr, which is close to what is achievable from a single plate. The long tail of objects with proper motions larger than about $80 \mathrm{mas} / \mathrm{yr}$ are real proper motion objects.

One problem which has arisen with the stacked data concerns star/galaxy separation. Fig. 4 shows a traditional plot of image area $\mathrm{A}$ against maximum intensity $\mathrm{T}$. For $\mathrm{T}$ values greater than about 200 , the stellar locus is reasonably well defined, but fainter than this discrimination is not possible. This limit is relatively speaking much worse than for a single plate, and seems to occur for two reasons. Firstly, the proportion of stars to galaxies decreases rapidly beyond $B_{j}$ $=21$, making the stellar locus hard to identify, and secondly as galaxies become fainter they become smaller, and with the rather large image size associated with Schmidt plates, the average galaxy soon becomes effectively unresolved. This means that projects will probably have to be designed in such a way that they do not rely heavily on morphological classification. 
PIXADO Blue

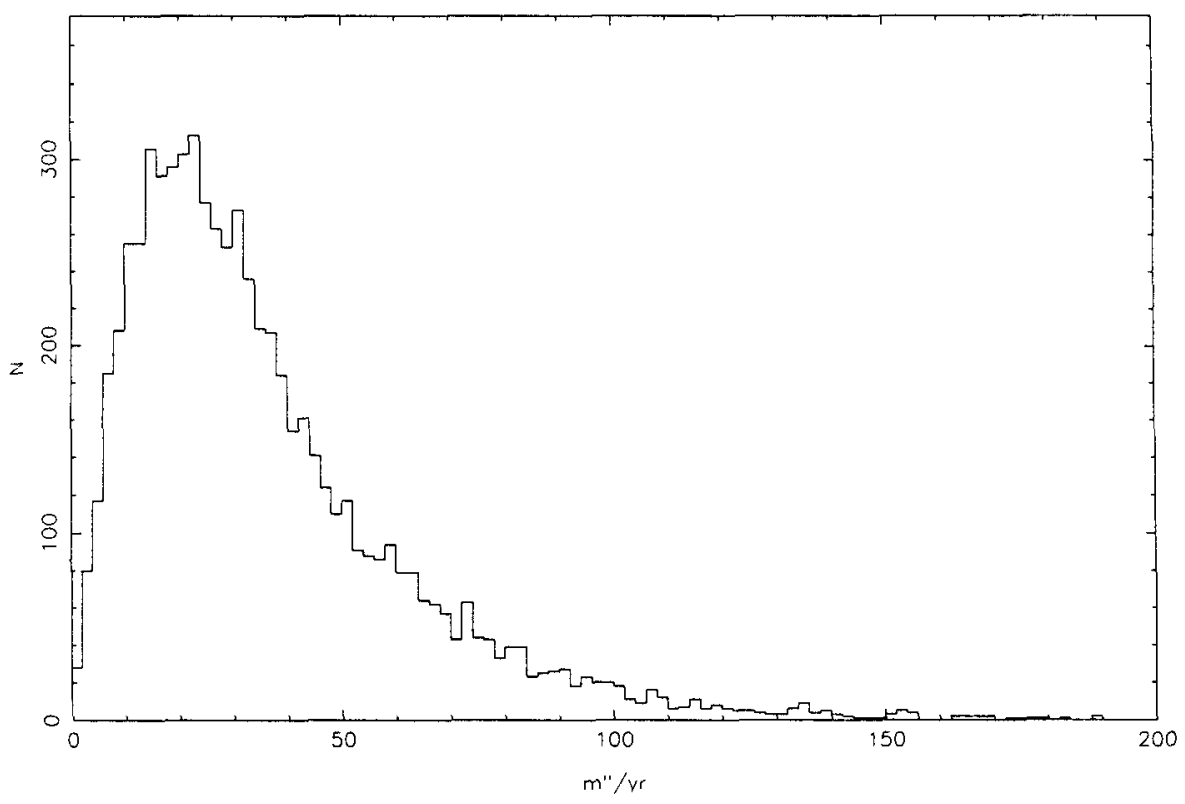

Figure 3. Histogram of proper motions from the Blue stack.

PIXRG1X

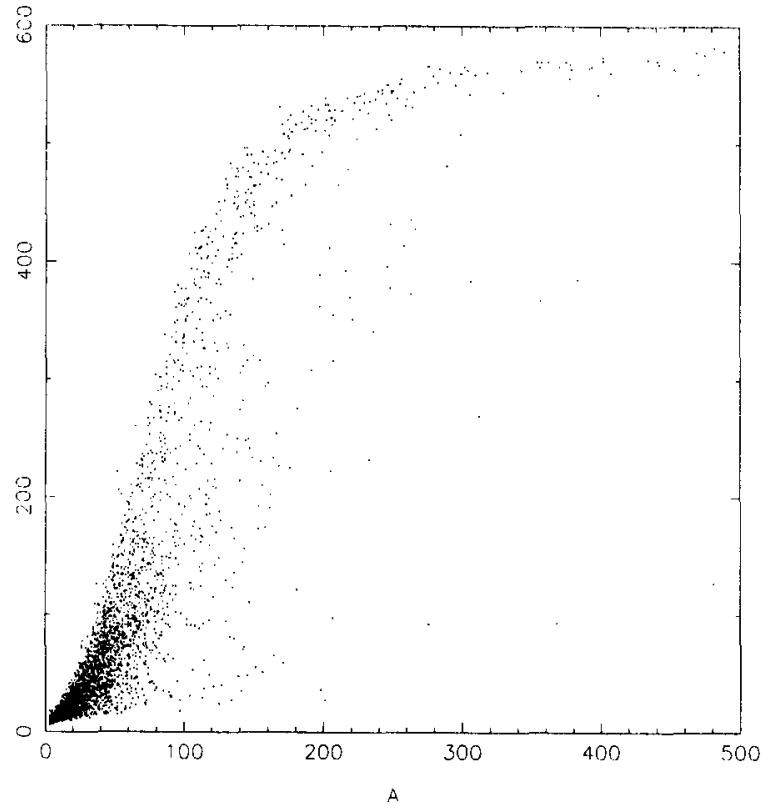

Figure 4. Plot of Area (A) against maximum density ( $\mathrm{T}$ ) for images near the centre of the blue stack. 


\section{Searches for Brown Dwarf Stars and Quasars}

The availability of a dataset with the limits outlined in Table 1 over 25 square degrees opens up a whole new range of possibilities. Large scale galaxy clustering projects in this new regime are clearly a very attractive possibility, but in the first instance the extension of some more straightforward stellar survey work has been carried out. The dataset is ideal for a number of such projects, including surveys for cool white dwarfs, unevolved halo stars and halo white dwarfs. However, the first project to be carried out was a survey for low mass stars or brown dwarfs.

The search for low mass stars followed the lines of the survey by Hawkins \& Bessell (1988), but using the deep $R$ and I stacks. In the first instance all objects with $R-I>2.5$ and $I<20.0$ were chosen in the central 25 square degrees. To eliminate spurious objects, the I stack was split into two halves containing early and late plates, and candidates were required to be detected on each of these substacks. This had the additional advantage that proper motions could be measured for all candidates. Although it was not included as a requirement, it turned out that all objects satisfying the above criteria were also detected on the $\mathrm{R}$ stack. This selection procedure resulted in a sample of around 250 objects which satisfied the criteria. These included all the known red stars in the field, as published by Hawkins \& Bessell (1988), and there was excellent agreement between both colours and proper motions for the two sets of measures. To date, the reddest known dwarfs have $R-I \approx 3.0$ in the photographic system, and there are only two or three such stars known. The survey based on the stack revealed 45 objects with $R-I>3.0$, and the reddest objects had $R-I \approx 4$ and $I \approx 20$, far redder than any known dwarfs. The spectrum of one star with $R-I=3.8$ is shown in Fig. 5. The molecular bands, especially TiO and V0 are the strongest so far observed, and the strong potassium line suggests that this is the coolest dwarf so far discovered. The proper motion measures may be used in conjunction with an assumed tangential velocity to estimate the luminosity, which comes out to be $\mathrm{M}_{R}=22.5$, some 3 magnitudes less luminous than stars such as LHS2924. There seems to be a strong case that this object is a brown dwarf.

Another early project has been the search for faint high redshift quasars. The most successful search procedure to date has involved looking for unusual coloured objects, with spectroscopic follow up. This method however is very inefficient as many candidates must be observed to find one quasar. Using yearly substacks it has been possible to combine colour information with variability measures along the lines described by Hawkins \& Véron (1993) to find faint high redshift quasars with a much higher success rate. Fig. 6 shows the light curves which led to the discovery of two faint high redshift quasars from the stack.

At the moment only a small number of test projects have been completed with the stacked data, but it seems reasonable to hope that a very wide variety of investigations will be extended into a completely new domain using the digitally stacked Schmidt plates. 

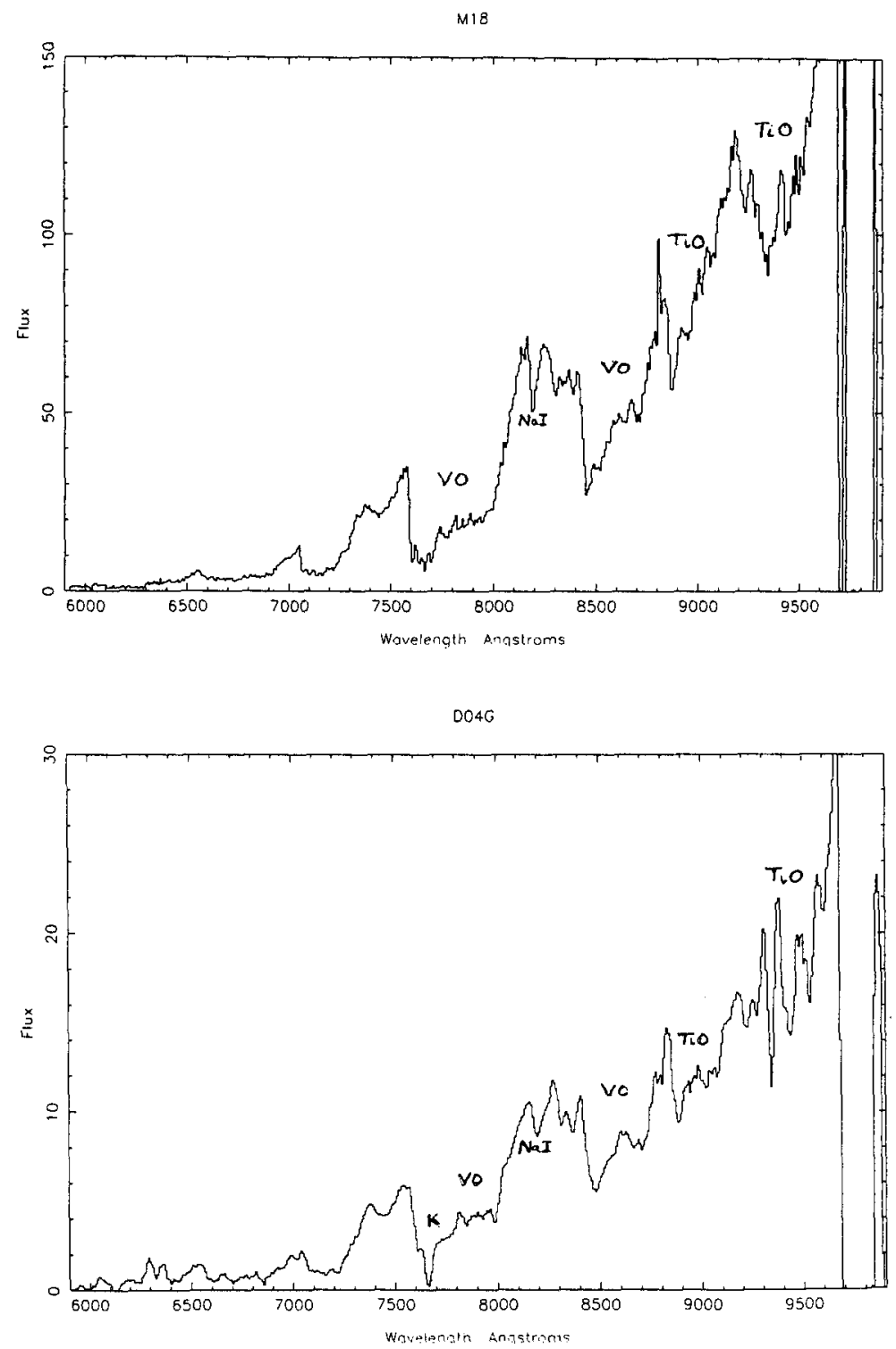

Figure 5. Spectra taken with EFOSC on the ESO $3.6 \mathrm{~m}$ for (top) one of the coolest dwarf stars currently known, and (bottom) a very cool star discovered from the stacked data. 


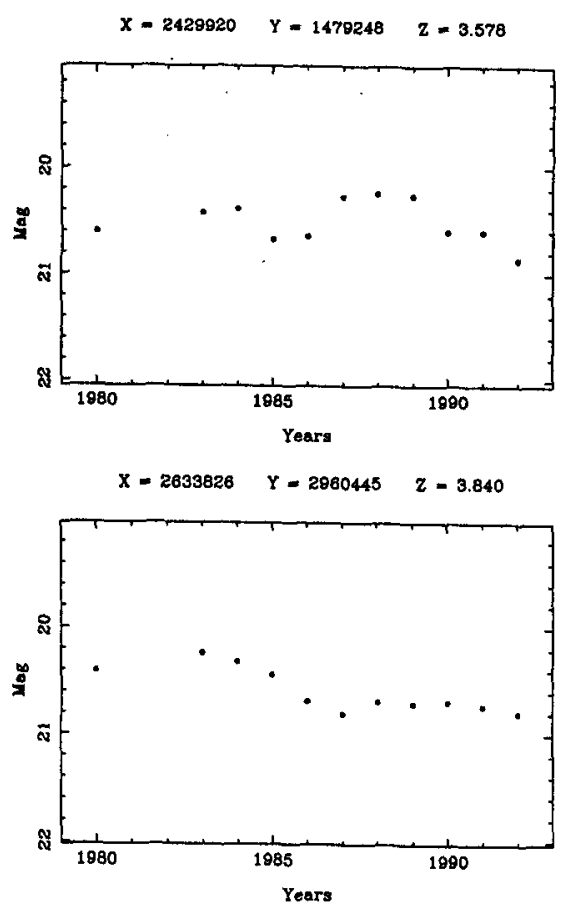

Figure 6. Light curves of two high redshift quasars discovered from variability from substacks of the IIIa-F plates.

\section{References}

Hawkins M. R. S. \& Bessell M. S., 1988, MNRAS, 234, 177

Hawkins M. R. S. \& Véron P., 1993 MNRAS, 260, 202

\section{Discussion}

Tsvetkov: How many plates do you have in your digitized survey and is it possible to have access to your data, and to the digitized COSMOS plates in general?

Hawkins: There are 65 plates in $B_{j}$ and $R$ pass-bands, 30 plates in $I$ and small numbers of $U$ and $V$ plates. Access to the plates would be by negotiation. 